\title{
Estimación del gasto total (TOTEX) de empresas de distribución mediante modelos de red de referencia y de fronteras de eficiencia
}

\author{
Carlos A. García-Montoya ${ }^{1}$, Jesús M. López-Lezama² y Tomás Gómez San Román ${ }^{3}$ \\ (1) Facultad de Minas, Universidad Nacional de Colombia Sede Medellín, Carrera 80 No 65-223, Medellín, Colombia \\ (correo-e:cgarciamo.@unal.edu.co). \\ (2) Departamento de Ingeniería Eléctrica, Universidad de Antioquia, Calle 67 No. 53-108, Oficina 19-437, Medellín, \\ Colombia (correo-e: jmaria.lopez@udea.edu.co) \\ (3) Universidad Pontificia Comillas, Instituto de Investigación Tecnológica, C. Alberto Aguilera 23 Madrid-28015, Madrid, \\ España (correo-e: tomas.gomez@comillas.edu)
}

Recibido Abr. 1, 2019; Aceptado Jun. 5, 2019; Versión final Jul. 30, 2019, Publicado Feb. 2020

\begin{abstract}
Resumen
Se desarrolla una metodología de estimación del gasto total, TOTEX, que permite a las empresas de distribución cuantificar los posibles márgenes de mejora para cumplir con los requisitos regulatorios. La estimación del TOTEX asociado con la actividad de distribución de electricidad es un problema dinámico debido a las condiciones cambiantes de su entorno económico y regulatorio. En consecuencia, las empresas de distribución requieren herramientas que les permitan enfrentar estos desafíos. La metodología propuesta en este trabajo integra los modelos de red de referencia y de fronteras de eficiencia. El uso de la metodología se ilustra en un sistema de distribución con aproximadamente 231000 clientes. Las pruebas muestran que la metodología desarrollada es adaptable al entorno cambiante, ya que puede integrar los problemas tecnológicos y de mercado.
\end{abstract}

Palabras clave: gasto total; TOTEX; distribución de electricidad; análisis de fronteras; modelos de red; fronteras de eficiencia

\section{Total expenditure estimation (TOTEX) of electricity distribution companies by means of reference network and of efficiency frontier models}

\begin{abstract}
A methodology for the estimation of total expenditure (TOTEX) that permits distribution companies to quantify the possible improvement margins to accomplish regulatory requirements. is proposed. TOTEX estimation, associated with the electricity distribution activity is a dynamic issue due to the changing conditions of its economic and regulatory environment. Consequently, distribution companies require tools that allow them to face these challenges. To this aim, the proposed methodology integrates reference network and efficiency frontier models. The use of the methodology is illustrated in a distribution system with approximately 231000 clients. Tests show that the proposed methodology can be adapted to the changing environment since it can integrate market and technological issues.
\end{abstract}

Keywords: total cost; TOTEX; electricity distribution; frontier analysis; network models; efficiency frontier 


\section{INTRODUCCIÓN}

El negocio de distribución de energía eléctrica se ve constantemente sometido a cambios regulatorios que propenden por un mejor desempeño en términos económicos y técnicos. Estos cambios se ven plasmados en mecanismos regulatorios que, a pasos incrementales, exigen mejorar la eficiencia de los distribuidores, de cara a transmitir esas eficiencias logradas a los clientes o usuarios finales (García-Montoya y López-Lezama, 2017). Bajo estas condiciones, las empresas de distribución operan en busca de lograr mayores márgenes de eficiencia, así como mejorar sus resultados económicos. Desde otro ángulo, los reguladores buscan constantemente diseñar mecanismos adecuados a la naturaleza de un negocio en constante cambio, pero que además garanticen una remuneración adecuada a las empresas por su servicio (Joskow, 2008, 2014). La remuneración por el servicio prestado del distribuidor está representada por un TOTEX (gastos totales), el cual a su vez está constituido por dos componentes principales: el CAPEX (los costos de capital) y el OPEX (los costos de operación). De manera general, el CAPEX representa el reconocimiento de los activos productivos necesarios para la distribución de energía y el OPEX corresponde a los recursos asociados a las actividades de administrar, operar y mantener el sistema (Gómez, 2013). Ambos componentes de la remuneración son constantemente revisados en busca de los valores eficientes, y adecuados, que garanticen un servicio óptimo, económicamente viable y sostenible.

Desde la perspectiva del regulador, se realizan estudios que buscan identificar la forma como se desarrollan mecanismos adecuados para incentivar la eficiencia, de cara a mejorar el servicio prestado dentro de niveles adecuados de costo (Jamasb y Pollitt, 2003; Joskow, 2007). En respuesta a esto, los distribuidores igualmente realizan ingentes esfuerzos para optimizar procesos, mejorar la calidad del servicio prestado y dimensionar de manera óptima la infraestructura necesaria para la actividad de distribución de energía (Gómez et al., 2012). Es aquí donde se evidencia la necesidad de que las empresas de distribución dispongan de herramientas aplicables a la planeación, de modo tal, que puedan identificar las mejoras o posibles brechas de eficiencia, tanto para el CAPEX como para el OPEX. La identificación de estas brechas permite mejorar los costos del operador y lograr un impacto positivo en el costo de la energía, sin comprometer la sostenibilidad de las empresas ni desmejorar la calidad del servicio.

Para el cálculo de un TOTEX óptimo y eficiente, se desarrolla una metodología integrada por los Modelos de Red de Referencia (RNM por sus siglas en inglés) y los Modelos de Fronteras de Eficiencia (EFM por sus siglas en inglés). Estas metodologías se han empleado en diversos mecanismos regulatorios (Jamasb y Pollitt, 2003, 2008). Si bien los RNM y EFM representan dos visiones diferentes, ambos se pueden integrar en una sola metodología que permita calcular el CAPEX y OPEX óptimos y eficientes de un grupo de empresas de distribución. Con la metodología integrada propuesta en este trabajo, las empresas de distribución pueden identificar las brechas de mejora a las cuales pueden llegar de acuerdo con un grupo de empresas que desarrollan su actividad económica bajo las mismas condiciones regulatorias; incluso teniendo en cuenta, y respetando, las características de los mercados que atienden. Adicionalmente, por medio de la integración de ambas metodologías, se logra un enfoque de solución del problema que permite identificar de manera holística el desempeño económico de la empresa junto con el impacto de posibles cambios en la infraestructura, considerando sus respectivos costos asociados. Todo esto, de cara a disminuir la incertidumbre existente durante el desarrollo del negocio debido al impacto del crecimiento del mercado, como también a los cambios tecnológicos y regulatorios.

\section{REGULACIÓN Y ESTIMACIÓN DE INGRESOS}

Desde hace casi cuatro décadas, se ha presentado un profundo cambio en diferentes mercados monopólicos, entre ellos el energético, pasando de ser negocios predominantemente de propiedad del Estado, para abrir las puertas a la inversión privada y convertirlos en mercados regulados (Joskow, 2008). La base principal del cambio de estos mercados se fundamentó en la necesidad de obtener precios justos por la prestación del servicio, aumentar la confiabilidad del sistema de distribución, incluir mecanismos antimonopolio y mejorar el servicio prestado a los clientes (Gómez, 2013). Estos aspectos en un mercado monopólico se encuentran ausentes considerando la falta de competencia, especialmente porque es esta la encargada de estimular a las empresas por mejorar su servicio y mantener precios que responden a la competencia del mercado atendido. Asunto que no sucede en la distribución de energía eléctrica dada su condición de monopolio, razón por la cual requiere convertirse en un negocio regulado. Bajo este contexto, la necesidad de estimar los costos de las empresas de distribución está íntimamente relacionada con el diseño de la regulación que determinan los entes reguladores. En esta sección se describe la evolución de las metodologías en materia de regulación y estimación de ingresos de empresas reguladas, observando algunas de sus fortalezas y debilidades.

\section{Regulación de Empresas de Distribución de Energía}

Los elementos hasta ahora citados cobran relevancia al momento de desarrollar un marco regulatorio exitoso y adecuado, de modo tal que este logre estimular la mejora en la calidad del servicio prestado por las 
empresas, obteniendo precios justos tanto para el consumidor final y una remuneración razonable para la empresa de distribución, obviamente, sin poner en riesgo la viabilidad financiera de la misma. Considerando la importancia de garantizar la viabilidad y sostenibilidad de las empresas, es importante determinar de manera adecuada los ingresos justos de las empresas de distribución. De cara a cumplir con estas premisas, se han desarrollado diferentes formas de determinar la remuneración de la empresa regulada.

Los primeros mecanismos regulatorios de empresas de distribución de energía remuneraban la totalidad de los costos en los que incurría la empresa, de modo que ésta no asumía ningún riesgo asociado con la demanda final esperada, bien fuera por los costos en que se incurría en la prestación del servicio o por el límite en las inversiones a realizar en los activos. Además, no se contaba con metas en términos de calidad del servicio que estimularan la mejora del desempeño. Esto se conoce como regulación por tasa de retorno (en inglés: rate-of-return). Obviamente, el reconocer en su totalidad los costos e inversiones realizadas, no genera ningún estímulo para que la empresa emplee de manera eficiente sus recursos buscando reducir los costos e inversiones. Como resultado, se obtienen efectos contraproducentes, que conllevan a grandes deficiencias en la regulación e ineficiencias operativas.

Dadas las debilidades observadas en la regulación por tasa de retorno, se desarrollaron metodologías que incentivaran la eficiencia de las empresas, tanto desde el punto de vista económico como en términos de los parámetros del servicio prestado. De esta forma surgió la regulación basada en incentivos. Este tipo de regulación, a diferencia del rate-of-return, define un ingreso estimado para el periodo regulatorio completo. Esto permite e incentiva a las empresas hacia la reducción de costos con miras a mejorar sus ganancias. Una vez terminado el periodo regulatorio, se revisan los componentes del ingreso aprobado y se ajustan según los resultados y la respuesta de las empresas al mecanismo regulatorio. Dos esquemas de regulación basados en este principio son price cap y revenue cap. El enfoque price cap define un precio máximo para el producto vendido, en este caso la energía, mientras el revenue cap define un ingreso máximo anual. Mayores detalles sobre ambos esquemas, pueden ser consultados en (Gómez, 2013). Pese a las fortalezas de la regulación basada en incentivos, existen debilidades tales como la posibilidad del deterioro en la calidad del servicio prestado, ocasionado por el incentivo enfocado en reducción de costos, lo cual puede afectar la disponibilidad de recursos para la atención del servicio. Como solución a esta situación, el regulador debe definir, de manera adecuada, mecanismos que penalicen a las empresas por incumplir con los límites de calidad del servicio exigidos (Fumagalli et al., 2007).

Un último grupo de metodologías regulatorias fundamenta su desarrollo en la comparación. Estas son denominadas Benchmarking (Jamasb \& Pollitt, 2003). Este tipo de mecanismos regulatorios, comparan un grupo de empresas respecto a un modelo, permitiendo determinar las potenciales mejoras que el distribuidor puede lograr (Cossent, 2013). Como parámetro de comparación pueden emplearse diferentes referentes: por una parte, están los modelos obtenidos mediante modelos econométricos, paramétricos o no paramétricos y, por otra parte, están los denominados Norm o Reference Model Methods (Jamasb y Pollitt, 2008) los cuales se emplean en la construcción de una empresa ideal que sirve como referencia para la determinación de la remuneración eficiente. Una desventaja de los métodos econométricos radica en la necesidad de gran cantidad de información, que no siempre está disponible para el regulador. Además, desacoplar los ingresos de los costos reales, puede ocasionar problemas financieros a las empresas dado que no garantiza unas ganancias que viabilicen la operación.

Por otra parte, el Norm Model Method emplea información disponible de las empresas para construir una empresa modelo considerando los parámetros óptimos definidos, entre la calidad del servicio prestado y atención de la demanda futura. Además, estos permiten hacer una estimación del efecto que puedan tener cambios tecnológicos sobre el desempeño de las compañías. Las diferentes metodologías de remuneración expuestas, permiten una adaptación de los diferentes mecanismos según el entorno bajo el cual se implementan. De acuerdo a esta evolución, el éxito de la regulación se encuentra en lograr un balance entre la eficiencia y la sostenibilidad del negocio, buscando el nivel de calidad del servicio adecuado, obviamente, considerando la remuneración justa de esta actividad económica.

\section{Estimación de Ingresos de Empresas de Distribución de Energía Eléctrica}

La remuneración de la distribución de energía eléctrica calcula el reconocimiento en dos componentes: OPEX y CAPEX, los cuales sumados componen el TOTEX. Además de estos, se reconoce una componente adicional, bien sea positiva o negativa, correspondiente a los incentivos logrados de acuerdo a los mecanismos regulatorios propios del mercado. En conjunto, OPEX y CAPEX, permiten estimar el nivel eficiente de ingresos esperados de una empresa de distribución. Este valor es de utilidad a la empresa para validar el nivel de retorno, posible y alcanzable, al ejecutar la planeación de las necesidades de inversión. Además, es posible estimar potenciales reducciones de costos y contar, de manera implícita, con el valor de ingresos eficientes esperados para un periodo regulatorio específico. Este valor, finalmente, refleja el nivel 
mínimo esperado de ingresos por la prestación del servicio. La cuantificación del TOTEX puede realizarse por medio de diferentes metodologías (Cossent, 2013). En (Miri et al., 2017) se presenta, por ejemplo, una regulación separada de OPEX y CAPEX para empresas de distribución que tiene en cuenta los plazos de gestión de activos. Los autores proponen un nuevo esquema de incentivos y penalizaciones para regular de manera eficiente la calidad de la red a un nivel deseable aplicado al caso iraní. En la literatura también se reportan diferentes formas de calcular el OPEX para un conjunto de empresas y luego particularizar el valor para una en específico (Cossent, 2013).

En este trabajo se emplean métodos de regresión multivariada que permiten, considerando un conjunto de variables explicativas, lograr una estimación de costos medios o característicos para un conjunto de empresas (Coelli et al., 2005). Si se desea encontrar un nivel de eficiencia en términos de costos, también para un grupo de empresas, se requiere de los modelos de fronteras de eficiencia. Estos son modelos paramétricos que hallan un límite óptimo para los costos de las empresas, empleando información histórica de las mismas (Kumbhakar y Lovell, 2000). Otras técnicas que pueden emplearse para el cálculo del OPEX y que han sido desarrolladas desde el mundo ingenieril, son los llamados modelos de red y/o algoritmos de optimización de infraestructura que calculan tanto el OPEX como el CAPEX, respectivamente. Dentro de estas técnicas, los RNM son usados especialmente para el cálculo del CAPEX óptimo, metodología que también es empleada en planeación de empresas y en el diseño de mecanismos regulatorios basados en la búsqueda de una infraestructura óptima para la prestación del servicio (Cossent, 2013). En (Gómez et al., 2011) se presenta el papel que la regulación española otorga a los RNM para el cálculo de los ingresos regulados de las empresas de distribución y se describen los detalles de datos de entrada del modelo y resultados proporcionados por el mismo. Las técnicas citadas para el cálculo de las componentes del TOTEX, han sido empleadas principalmente en el desarrollo de mecanismos regulatorios, especialmente bajo esquemas de benchmarking. Una clasificación detallada de las diferentes metodologías de referenciamiento puede encontrarse en (Cossent, 2013).

Dadas las condiciones expuestas en esta sección, tanto entes reguladores como empresas de distribución, emplean recursos en el desarrollo constante de metodologías que permiten estimar los niveles óptimos o eficientes, tanto de OPEX como de CAPEX. No obstante, en la literatura no se observa una metodología que permita a los distribuidores identificar las brechas o potenciales mejoras con miras al cumplimiento de las exigencias regulatorias. Es así, como en el presente trabajo, se integran dos metodologías con el objetivo de lograr un nivel óptimo de CAPEX que garantice la atención del servicio y un OPEX eficiente que considere las particularidades de un mercado de energía. La suma de ambos componentes permite determinar el máximo nivel de eficiencia que puede lograr el distribuidor para la atención de un mercado específico, considerando variables como: entorno, topología real, costos por unidad constructiva, objetivos o metas de calidad del servicio y variables de dimensión del mercado atendido, entre otros. Adicionalmente, desde la planeación de la empresa, se puede identificar el efecto estimado futuro de algunas variables de entorno.

\section{METODOLOGIA}

De acuerdo con el problema planteado, las empresas de distribución requieren de herramientas que les permitan evaluar adecuadamente los ingresos estimados anuales $y$, basados en esta expectativa, evaluar la viabilidad de las inversiones y el costo operativo frente a este valor. En este trabajo se desarrolla una metodología para la estimación del TOTEX, de acuerdo con las expectativas mencionadas. Para lograr este objetivo, se emplean dos metodologías: un modelo de red de referencia para el cálculo del CAPEX y un modelo de frontera de eficiencia para el cálculo del OPEX eficiente. Luego, tal como se presenta en la ecuación (1), los valores resultantes se suman para lograr el TOTEX esperado.

$$
\text { TOTEX }=\text { CAPEX }+ \text { OPEX }
$$

Con las metodologías para calcular el TOTEX, se logra identificar el efecto de los cambios tecnológicos sobre los costos de CAPEX gracias a las características propias del modelo de red de referencia. La estimación por medio de esta metodología le permite al operador de red obtener los costos de infraestructura necesaria considerando la red óptima para la atención de los clientes, incluyendo una estimación del costo adicional por la atención de nuevos clientes o crecimiento de la demanda, entre otras posibilidades.

Por otra parte, el uso de modelos de frontera de eficiencia para el cálculo del OPEX, permite identificar el nivel eficiente de costos del operador de red, con la ventaja de incluir los costos de AOM (Administración, Operación y Mantenimiento) que por otros métodos de estimación no se podrían calcular con una visión holística del negocio o con visión integral de empresa como unidad productiva. Finalmente, el cálculo del TOTEX para la empresa de distribución, mediante la metodología desarrollada, permite que existan trade-offs entre los costos de operación e inversiones. De esta forma, el distribuidor está en libertad de decidir que tecnología o metodología emplear en la actividad de distribución, buscando el máximo nivel de eficiencia posible según su experticia y conocimiento del mercado y la infraestructura que opera. 
La metodología propuesta fue validada con datos de empresas reales. Adicionalmente, fueron simulados algunos escenarios de prueba sobre el caso de estudio, considerando las señales de crecimiento de la demanda indicadas por el ente planeador colombiano (UPME -Unidad de Planeación Minero Energética) y el valor medio de clientes nuevos por año. A continuación, se presentan tanto los fundamentos correspondientes a los modelos de frontera de eficiencia paramétricos, como los modelos de red de referencia.

\section{Modelos de Red de Referencia - RNMs}

Los RNMs son modelos de optimización de las redes de distribución que permiten construir redes de referencia que pueden ser usadas tanto para la planeación de las empresas como para la estimación de costos óptimos del distribuidor, obteniendo tanto el CAPEX como el OPEX (Jamasb y Pollitt, 2008). Dado que en este trabajo se desea calcular un OPEX que incluya de manera general la empresa de distribución, el OPEX es calculado con otra metodología y se emplea solo el CAPEX obtenido mediante un RNM.

Los RNMs pueden ejecutarse bajo diferentes enfoques. Un enfoque desde cero o modo greenfield y un modelo de planeación de expansión o modo brownfield. El modo greenfield construye las redes necesarias para la prestación del servicio partiendo de la localización de las cargas georeferenciadas y la ubicación de unas subestaciones de distribución, siempre en búsqueda de la red óptima bajo criterios mínimos de calidad del servicio y de pérdidas del sistema. En la Fig. 1 puede apreciarse un esquema general del proceso llevado a cabo por el modelo de red de referencia para la construcción de la red. Mayor detalle sobre los RNMs puede encontrase en (Domingo et al., 2011).

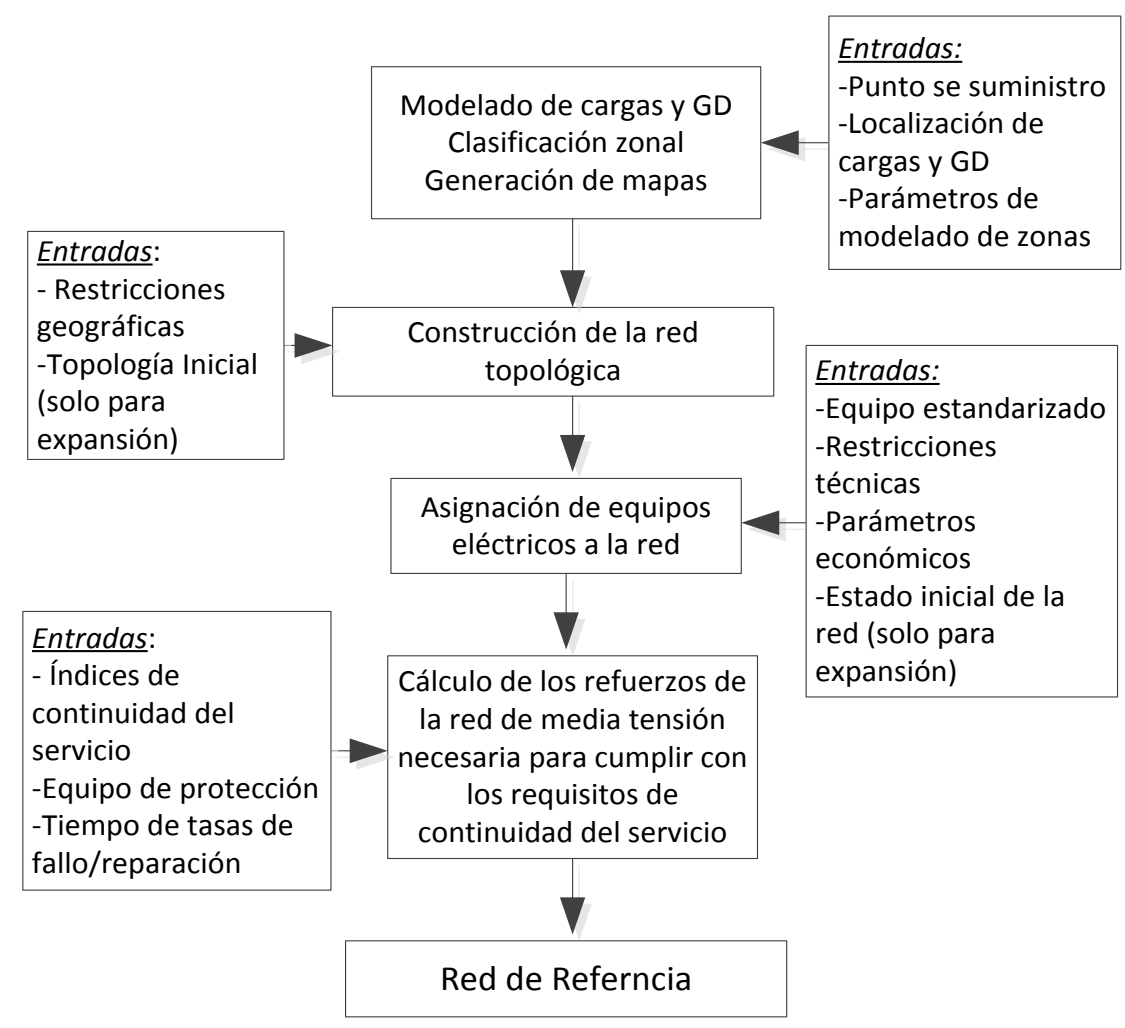

Fig. 1: Diagrama del proceso computacional desarrollado por un RNM (García et al., 2016).

Una de las mayores fortalezas de esta metodología radica en la capacidad de evaluar el impacto de los cambios tecnológicos sobre la infraestructura de distribución, obteniendo los costos de capital que pueden generar el ingreso de nuevas tecnologías. En (Cossent et al., 2011; García et al., 2016) se reporta el uso de la RNM en la estimación del impacto de la penetración de generación distribuida fotovoltaica (GDFV) en Colombia, España, Alemania y Holanda. Estos trabajos evalúan diferentes tipos de infraestructura, pasando desde una topología enmallada, como es natural en los sistemas de distribución europeos, hasta un sistema altamente radial como es el caso colombiano. Dentro de estos trabajos se hace un análisis particular de diferentes niveles de penetración de GDFV bajo diferentes escenarios, incluyendo el crecimiento de demanda o sin ella.

En el presente trabajo se empleó la RNM en sus dos modos, greenfield y brownfield. Inicialmente se suministra la localización de las cargas existentes y se le permite al modelo crear, bajo el modelo greenfield, las redes necesarias para la atención del servicio, limitando al modelo la posibilidad de construir nuevas subestaciones. 
Con esta restricción se busca que la topología de la red sea similar al tipo de construcción en el mercado colombiano, el cual es predominantemente radial. Luego, empleando el modelo brownfield, se evalúan diversos escenarios de crecimiento como ya se mencionó al inicio de la sección. Esto permite que se identifique el efecto del crecimiento de la demanda y el crecimiento de los clientes atendidos sobre el CAPEX.

\section{Modelos de Fronteras de Eficiencia}

Una vez calculado el CAPEX, la segunda parte de la metodología de estimación corresponde al cálculo del OPEX mediante el uso del análisis de fronteras de eficiencia. La metodología de cálculo desarrollada en el presente trabajo está basada en Frontier Benchmarking y Norm Models. El Frontier Benchmarking se diferencia de los Norm Models particularmente por su enfoque y origen, el cual no es ingenieril sino econométrico. Desde este punto de vista, el Frontier Benchmarking se fundamenta en toda una teoría relacionada con la medición de eficiencia y productividad. El resultado obtenido, desde la actividad económica de una empresa, se ve influenciado por la tecnología de producción y algunos agentes exógenos que afectan el proceso. Es así como se llega a la eficiencia productiva, que corresponde a la capacidad de la empresa de producir, una unidad o un producto, a un costo determinado.

Desde el punto de vista microeconómico existen dos tipos de eficiencia: la eficiencia técnica y la asignativa. La eficiencia técnica es definida como la habilidad de obtener máximo producto bajo unos niveles definidos de insumos y tecnología de producción, midiendo la habilidad de usar bajos recursos para producir un número de productos determinado. Por otro lado, la eficiencia asignativa es la habilidad de combinar de manera óptima los productos e insumos disponibles (Pahwa et al., 2002). El nivel óptimo de eficiencia puede definirse como el costo u otro objetivo que la compañía tenga definido. De esta forma surgen dos tipos de modelos, clasificados de manera general como modelos de frontera de costos y de producción (Melo y Espinosa, 2005). El modelo de frontera de costo es el empleado en el presente trabajo, por su capacidad de representar una frontera de costo eficiente de un grupo de empresas. Los modelos de frontera de producción no son tratados en este trabajo dado que no son utilizados dentro de la metodología. Mayores detalles sobre los modelos de frontera de producción y costo, puede ser consultados en (Coelli et al., 2005).

La estimación de los modelos de fronteras de eficiencia puede realizarse por medio de dos metodologías: primero el denominado análisis envolvente de datos (DEA por su sigla en inglés) cuya estimación está basada en programación lineal de forma no paramétrica y, segundo, el denominado análisis de fronteras estocásticas (SFA por su sigla en inglés) que está fundamentado en métodos econométricos. En (Navarro et al., 2019) se presenta una aplicación del DEA en el análisis de la eficiencia del sector eléctrico en México. Se presentan resultados que evidencian un marcado contraste en la eficiencia según la región estudiada. Los autores concluyen que, si bien se ha contado con la capacidad de abastecer la demanda, es necesario continuar con estudios de esta índole. La principal ventaja del DEA consiste en que no está basado en el conocimiento de la función de producción. Por otro lado, el SFA permite obtener una forma funcional que representa la frontera. El potencial de los métodos de medición de eficiencia radica en que hacen posible comparar un grupo de empresas con mercados y características diferentes pero que desarrollen la misma actividad económica, permitiendo una regulación o comparación equitativa para las mismas (Rodríguez et al., 1999). También han surgido técnicas híbridas que permiten integrar las ventajas de las técnicas paramétricas y no paramétricas como la propuesta en (Kuosmanen et al., 2013). La metodología basada en SFA fue elegida por que su enfoque paramétrico permite identificar el efecto que tienen las variables postuladas como explicativas sobre los costos de las empresas de distribución y por su versatilidad, pues permite realizar análisis de sensibilidad sobre el efecto de diferentes variables y proponer diferentes modelos.

La aplicación de SFA se realizó con una muestra de 30 datos de empresas de distribución en Colombia, constituyendo una muestra transversal. Las variables empleadas en el modelo ajustado son: energía trasmitida por el operador de red, número de clientes y kilómetros de red totales del sistema de distribución. La selección de estas variables se fundamentó en la estimación de un modelo de regresión donde fueron encontradas como explicativas (es decir, con un peso significativo dentro del modelo). Finalmente, estas variables se emplearon para el ajuste del modelo de frontera, con el cual se efectúa la estimación del OPEX eficiente. El modelo ajustado corresponde a un modelo Cobb-Douglas, el cual está representado en forma genérica en la ecuación (2). Donde el término de la izquierda corresponde al logaritmo natural de costos de la empresa $i$ y $X_{n i}$ corresponde a la variable $n$ de la empresa $i$. Por su parte los $\beta$ corresponden a los coeficientes que determinan el peso de la variable en la frontera. Para mayores detalles sobre la metodología de estimación puede consultarse (Kumbhakar y Lovell, 2000).

$$
\operatorname{Ln} Y_{i}=\beta_{0}+\sum_{n=1}^{N} \beta_{n} \operatorname{Ln} X_{n i}+v_{i}+u_{i}
$$


Concluida la estimación del modelo de frontera de eficiencia, se procede al cálculo del TOTEX estimado según los escenarios propuestos, los cuales se describen en la siguiente sección.

\section{RESULTADOS}

La metodología desarrollada fue empleada en un grupo de empresas de distribución colombianas. En la Tabla 1 , puede apreciarse un resumen de la estadística descriptiva de los datos de las empresas empleadas. Se cuenta con una muestra con amplio rango en términos de: área de cobertura, cantidad de clientes, energía distribuida y las dimensiones del área de cobertura de las empresas.

Tabla 1: Estadística descriptiva de la muestra empleada

\begin{tabular}{|l|l|l|c|c|c|c|}
\hline \multicolumn{1}{|c|}{ Descripción } & \multicolumn{1}{c|}{ Variable } & \multicolumn{1}{c|}{ Unidad } & Media & Desv. est. & Mínimo & Máximo \\
\hline Área de cobertura & Área_C & Kilómetros cuadrados & 25,060 & 20,726 & 1,962 & 60,728 \\
\hline $\begin{array}{l}\text { Energía transportada por } \\
\text { el sistema }\end{array}$ & Energ_tran & GWh & 228 & 252 & 30 & 786 \\
\hline Clientes & Clien & - & 733,870 & 682,845 & 155,127 & $2,117,000$ \\
\hline Kilómetros de red & Kms_Total & Kilómetros & 33,818 & 25,247 & 4,812 & 85,397 \\
\hline
\end{tabular}

Como se ha comentado, la metodología planteada calcula un OPEX por medio de un EFM y, para completar el TOTEX, emplea un RNM para la estimación del CAPEX. Previo a emplear el SFA para la obtención del modelo de frontera de eficiencia, con el objetivo de verificar la capacidad explicativa de las variables postuladas, se estimó un modelo de regresión multivariado. En la Tabla 2, pueden apreciarse los parámetros y las variables empleadas para obtener el modelo capaz de modelar los costos de las empresas de distribución. Esto considerando que la variable dependiente del modelo en este caso es el OPEX. De acuerdo con (2), las variables postuladas al modelo corresponden al logaritmo natural (Ln) de las variables originales. Por esta razón, las variables en las Tablas 2 y 3 se ven afectadas por el Ln. El modelo de regresión obtenido, logró un valor de $\mathrm{R}^{2}$ de 0.978 , lo cual indica que las variables explican adecuadamente los costos de las empresas consideradas en el estudio. En la Tabla 3, pueden apreciarse los parámetros del EFM que se obtuvo como característico de los costos de las empresas empleadas en el estudio. Estos parámetros corresponden al modelo con el cual se estiman los valores de OPEX de las empresas.

El caso de estudio empleado como ilustración en el presente trabajo corresponde a un sistema de distribución que atiende aproximadamente a 231,000 clientes y cuenta con 22,000 transformadores de distribución. Este caso de estudio cuenta con una combinación entre centros poblados urbanos y zona rural dispersa. Dicha combinación fue elegida intencionalmente con el objetivo de identificar el efecto de la dispersión de los clientes sobre la infraestructura, considerando una topología radial típica colombiana. Recopilada esta información, se procede a la ejecución de la RNM en modo Greenfield.

Tabla 2: Variables y parámetros del modelo de regresión

\begin{tabular}{|c|c|c|c|}
\hline Variable & Unidad & Coeficiente & Error standard \\
\hline Ln Energ_tran & GWh & 0.0635 & 0.0555 \\
\hline Ln Clien & - & 0.1404 & 0.0669 \\
\hline Ln Kms_Total & Kilómetros & 0.5485 & 0.0572 \\
\hline Constante & - & 3.9741 & 0.5064 \\
\hline
\end{tabular}

Tabla 3: Variables y parámetros del modelo de frontera estimado

\begin{tabular}{|c|c|c|c|}
\hline Variable & Unidad & Coeficiente & Error standard \\
\hline Ln Energ_tran & GWh & 0.0225 & 0.0494 \\
\hline Ln Clien & - & 0.2141 & 0.0756 \\
\hline Ln Kms_Total & Kilómetros & 0.5193 & 0.0529 \\
\hline Constante & - & 3.3762 & 0.4904 \\
\hline
\end{tabular}

Una vez se obtiene la red óptima generada por la RNM, se proyectaron los crecimientos de la demanda y clientes, con el objetivo de calcular el efecto sobre el CAPEX de esta variable. De esta forma, mediante el uso de la RNM se logra estimar el efecto real en términos de construcción de nuevas redes o refuerzos del sistema existente, que son requeridos por el ingreso de nuevos clientes y los incrementos en el consumo. Para simular 
estos escenarios, se emplea la RNM en modo brownfield. En este caso, la salida del modelo greenfield es la entrada de esta etapa. En esta misma etapa, con el objeto de plantear los escenarios estimados, se proyectan aleatoriamente los nuevos clientes en el espacio donde la empresa presta el servicio, además de proyectar el crecimiento de la demanda del $2 \%$ promedio anual, dato extraído de las proyecciones de demanda de la UPME (UPME, 2016).

El cálculo final del CAPEX se efectúa empleando el Valor Nuevo de Reemplazo (VNR) de la infraestructura óptima estimada por la RNM, multiplicado por la tasa de retorno definida por el regulador (CREG, 2015). Para el caso colombiano esta corresponde al 13.9\% antes de impuestos. Otras metodologías de estimación del valor de los activos se pueden emplear. En la Tabla 4, pueden apreciarse los resultados del cálculo del TOTEX eficiente estimado mediante la metodología propuesta, considerando el crecimiento de demanda de $2 \%$ anual y un ingreso 5000 clientes nuevos anuales. Los valores estimados de las variables se emplean para obtener un valor proyectado de OPEX según el EFM ya definido con anterioridad. Este valor sumado al CAPEX estimado da como resultado final el TOTEX esperado para cada escenario propuesto. Vale la pena indicar que los resultados obtenidos son de carácter ilustrativo para la presentación de la metodología

Tabla 4: Variables y parámetros del modelo de frontera estimado

\begin{tabular}{|c|c|l|l|l|c|c|}
\hline Proyección & $\begin{array}{l}\text { Incremento } \\
\text { demanda [\%] }\end{array}$ & $\begin{array}{l}\text { Número de } \\
\text { clientes }\end{array}$ & $\begin{array}{l}\text { Longitud de red } \\
{[\text { Km] }}\end{array}$ & $\begin{array}{l}\text { OPEX } \\
\text { [MCOP] }\end{array}$ & $\begin{array}{l}\text { CAPEX } \\
\text { [MCOP] }\end{array}$ & $\begin{array}{l}\text { TOTEX } \\
\text { [MCOP] }\end{array}$ \\
\hline 0 & 0 & 231,000 & 10,770 & 56,669 & 89,157 & 145,826 \\
\hline 1 & 2 & 236,000 & 11,035 & 57,678 & 91,603 & 149,281 \\
\hline 2 & 4 & 241,000 & 11,246 & 58,536 & 92,560 & 151,096 \\
\hline 3 & 6 & 246,000 & 11,438 & 59,339 & 93,741 & 153,080 \\
\hline
\end{tabular}

\section{DISCUSIÓN FINAL}

En este artículo se propone una metodología que permite a las empresas de distribución estimar tanto los costos operativos como los costos de capital eficientes requeridos para la prestación del servicio de energía eléctrica. La metodología presenta una alta adaptabilidad frente a los cambios tecnológicos a los que se ve sometido el sistema de distribución. Esto se logra por medio de la aplicación de la RNM. Esta metodología permite estimar las necesidades de modificación de infraestructura bajo diferentes escenarios, bien sea frente a crecimiento de la demanda, cambios tecnológicos o del modelo de negocio, como pueden ser: los cambios en los hábitos de consumo que afecten la demanda, la penetración de generación distribuida, la autogeneración, la respuesta de la demanda, entre otros. De cara a los desafíos regulatorios, esta metodología también les permite a las empresas de distribución evaluar su desempeño en términos de eficiencia en costos operativos, lo cual les facilita la definición de metas de optimización de costos de distribución mediante el uso del EFM.

En el presente trabajo se emplearon tres variables como explicativas de costos de las empresas de distribución. Estas se seleccionaron porque se observó que son las que mejor explican los costos para este grupo de empresas. No obstante, otras variables pueden ser consideradas, según sea el caso y dimensiones de la muestra de empresas que se tenga a disposición. La posibilidad de adaptarse a diferentes entornos, brinda a la metodología una amplia flexibilidad que permite su aplicabilidad en diferentes grupos de empresas y entornos económicos. La metodología propuesta puede ser aplicada desde diferentes ángulos: desde el punto de vista de las empresas de distribución esta puede considerarse una herramienta que facilite la definición de metas internas o una herramienta que permita afrontar las exigencias regulatorias en términos de mejora en eficiencia. Por otra parte, desde el punto de vista regulatorio, dadas las características implícitas que tiene la metodología, esta puede ser empleada para definir mecanismos regulatorios basados en benchmarking, fundamentadas en condiciones reales del entorno de las empresas, mejorando las proyecciones realizadas y considerando cambios tecnológicos. De esta forma se conjugan elementos que otras metodologías no han logrado involucrar de manera conjunta.

Dentro de la búsqueda constante por un costo de energía que sea sostenible desde el punto de vista económico, que cumpla con los estándares de calidad y la minimización del impacto tarifario debido al costo de distribución; esta metodología permite identificar los márgenes de eficiencia en costos y optimización de infraestructura que son deseables. Pese a que estos no siempre son alcanzables debido a que la infraestructura construida no siempre se puede reemplazar en el corto plazo, la aplicación de esta metodología permite que se identifiquen potenciales mejoras que gradualmente se pueden implementar, para con ellas lograr el balance óptimo que se desea. 


\section{CONCLUSIONES}

De acuerdo al trabajo presentado y a los resultados obtenidos, se pueden plantear las siguientes conclusiones principales:

1.- Se presentó una nueva metodología para el cálculo de TOTEX de empresas de distribución que les permite cuantificar los posibles márgenes de mejora para cumplir con los requisitos regulatorios. Esta metodología combina las fortalezas de los modelos de red de referencia y las fronteras de eficiencia. La aplicabilidad y efectividad de la metodología propuesta se evidenció mediante la aplicación a un sistema de distribución real.

2.- La metodología de cálculo de CAPEX y OPEX eficiente para redes de distribución propuesta en este artículo, puede ser aplicada a un conjunto de empresas de distribución heterogéneas que se enfrenten a realidades muy distintas desde el punto de vista de número de clientes, cantidad de energía transportada, kilómetros de red, área atendida (rural o urbana), etc.

3.- El uso combinado de modelos de red de referencia con fronteras de eficiencia para la estimación del CAPEX y OPEX le imprime a la metodología adaptabilidad a un entorno cambiante, ya que de manera natural puede integrar problemas tecnológicos y de mercado. Adicionalmente, la metodología puede aplicarse desde el punto de vista de las empresas de distribución o desde el punto de vista del regulador.

\section{AGRADECIMIENTOS}

Los autores expresan su agradecimiento a la Vicepresidencia Transmisión y Distribución Energía de Empresas Públicas de Medellín por su apoyo para el desarrollo de este trabajo, al Proyecto de Sostenibilidad de la Universidad de Antioquia, al Instituto de Investigación Tecnológica (IIT) de la Universidad Pontificia Comillas por el apoyo y recursos para la estancia doctoral y al Dr. Carlos Mateo Domingo por sus aportes en la comprensión y aplicación del RNM.

\section{REFERENCIAS}

Coelli, T.J., D.S.P Rao., C.J. O’Donnell y G.E Battese., An Introduction to Efficiency and Productivity Analysis (2. ${ }^{a}$ ed.), Springer Science \& Business Media (2005).

Cossent, R., Economic regulation of distribution system operators and its adaptation to the penetration of distributed energy resources and smart grid technologies, Universidad Pontificia Comillas, Madrid, España (2013).

Cossent, R., L. Olmos y otros tres autores, Distribution network costs under different penetration levels of distributed generation, DOI: 10.1002/etep.503, European Transactions on Electrical Power, 21(6), 1869-1888 (2011).

CREG., Resolución 095, Colombia (2015).

Domingo, C.M., T.G.S. Roman., A. Sanchez., J.P.P. Gonzalez y A.C. Martinez., A Reference Network Model for LargeScale Distribution Planning with Automatic Street Map Generation, DOI: 10.1109/TPWRS.2010.2052077, IEEE Transactions on Power Systems, 26(1), 190-197 (2011).

Fumagalli, E., I. Schiavo y F. Delestre., Service Quality Regulation in Electricity Distribution and Retail, Berlin, Heidelberg: Springer Science \& Business Media (2007).

García, C.A., T. Gómez y J.M. López-Lezama., Costos de distribución de energía eléctrica bajo la penetración de generación distribuida fotovoltaica, en XIII Jornada de distribución de energía eléctrica, ASOCODIS, Bogotá, Colombia (2016).

García-Montoya, C. A. y J.M. López-Lezama, Caracterización del Costo de Distribución de Energía Eléctrica Mediante Modelos de Fronteras de Eficiencia considerando un Indicador de Calidad del Servicio, DOI: 10.4067/S071807642017000200005, Inf. Tecnol., 28(2),37-46 (2017).

Gómez, T., C. Mateo, A. Sánchez, J. Reneses y M. Rivier, La retribución de electricidad en España y el Modelo de Red de Referencia, Estudios de Economía Aplicada, ISSN 1697-5731, 29(2), 1-24, (2011).

Gómez, T., Monopoly Regulation, en I. Pérez Arriaga (Ed.), Regulation of the Power Sector, DOI: 10.1007/978-1-44715034-3_4, 151-198. London, United Kingdom: Springer (2013).

Gómez, V.A., R., Peña y C. Hernández, Identificación y Localización de Fallas en Sistemas de Distribución con Medidores de Calidad del Servicio de Energía Eléctrica, DOI: 10.4067/S0718-07642012000200013, Inf. Tecnol., 23(2), 109-116 (2012)

Jamasb, T y M. Pollitt., International benchmarking and regulation: an application to European electricity distribution utilities, Energy Policy, DOI: 10.1016/S0301-4215(02)00226-4, 31(15), 1609-1622, (2003).

Jamasb, T y M. Pollitt., Reference models and incentive regulation of electricity distribution networks: An evaluation of Sweden's Network Performance Assessment Model (NPAM), DOI: 10.1016/j.enpol.2008.01.03, Energy Policy, 36(5), 1788-1801, (2008). 
Joskow, P.L., Chapter 16 Regulation of Natural Monopoly, en A. M. Polinsky \& S. Shavell (Eds.), Handbook of Law and Economics DOI: 10.1016/S1574-0730(07)02016-6, 2, 1227-1348, Elsevier, (2007).

Joskow, P.L., Incentive Regulation and Its Application to Electricity Networks, Review of Network Economics, ISSN: 14469022, 7(4), 1-14 (2008).

Joskow, P.L., Incentive Regulation in Theory and Practice: Electricity Distribution and Transmission Networks. Economic Regulation and Its Reform: What Have We Learned? University of Chicago Press, 291-344 (2014).

Kumbhakar, S y K. Lovell., Stochastic Frontier Analysis. Cambridge University Press (2000).

Kuosmanen, T., A. Saastamoinen y T. Sipiläinen., What is the best practice for benchmark regulation of electricity distribution? Comparison of DEA, SFA and StoNED methods, DOI: 10.1016/j.enpol.2013.05.091, Energy Policy, 61, 740750 (2013).

Melo, L y N. Espinosa., Ineficiencia en la distribución de energía eléctrica: una aplicación de las funciones de distancia estocástica, Revista ESPE, ISSN: 2631-2603, 23(49), 88-132 (2005).

Miri S.M., M.R. Haghifam, M. Zangiabadi y M. H. Bahmani, Designing a comprehensive separated regulation mechanism of distribution companies considering shot and long-term asset management, DOI: 10.1049/oap-cired.2017.1342, CIRED Open Access proceeding Journal, 1, 2674-2677 (2017).

Navarro J.C., O. Delfín y A. Díaz, La eficiencia del sector eléctrico en México 2008-2015, DOI: 10.1016/S02727757(01)00068-1. Análisis Económico, 85, 71-94 (2019).

Pahwa, A., X. Feng y D. Lubkeman., Performance evaluation of electric distribution utilities based on data envelopment analysis, DOI: 10.1109/TPWRS.2003.814835, IEEE Transactions on Power Systems, 17(3), 400-405 (2002).

Rodríguez, M., M. Rossi y C. Ruzzier., Fronteras de eficiencia en el sector de distribución de energía eléctrica: la experiencia sudamericana (UADE Textos de Discusión 15_1999 No. 15_1999), Instituto de Economía, Universidad Argentina de la Empresa (1999).

UPME, Proyección de demanda de energía eléctrica y potencia máxima en Colombia, Bogotá, Colombia (2016). 